\section{Quantitative Angiographic and Histopathologic Evaluation of Experimental Aneurysms}

The article of Bouzeghrane et $\mathrm{al}^{1}$ is a timely and surely important contribution to reaching standardized quality criteria for experimental aneurysm models and evaluation techniques. Nevertheless, the following comments should be added.

The authors of the above-mentioned article state that the lack of correlation between angiographic and histopathologic results is a major limitation to the validity of experimental aneurysm studies. This statement holds true if subjective estimation of aneurysm occlusion is used as in most of the cited literature. However, our studies have shown good correlation of angiographic and histopathologic occlusion rates by applying a new method of computerized quantification, first on 8 venous pouch bifurcation aneurysms in rabbits. ${ }^{2}$ Because this correlation reached statistical significance in a subsequent large postmortem series of 14 human aneurysms, ${ }^{3}$ the superior precision of computer-assisted quantification for evaluating angiograms and corresponding surface-stained ground sections from polymethylmethacrylate-embedded aneurysms has been validated.

Concerning the best suitable specimen preparation for histopathologic evaluation, the authors stated that cutting-grinding techniques with the devices in situ preclude advanced histotechniques such as immunohistochemistry. This limitation applies only to the past because currently several resin-embedding techniques and materials (eg, Technovit 9100 New; Heraeus Kulzer, Wehrheim, Germany) are available, in which denaturation of proteins is avoided by low polymerization temperatures $\left(\right.$ at $-20^{\circ} \mathrm{C}$ ). Therefore, the major advantage of histotechniques preserving the implants in situ to evaluate cell reactions at implant-tissue interfaces can now be combined with immunocytohistochemistry.

As a critique of the rabbit venous pouch bifurcation model, Bouzeghrane et $\mathrm{al}^{1}$ showed, in Table 4, that neck endothelialization was not described and thrombus organization in the aneurysm sac was not properly followed because only uncovered coils were found in the lumen and no fibrosis was visible between the loops of coils. However, all these features of aneurysm healing have been described in detail by our group ${ }^{2}$ and also by several other authors in rabbit venous pouch aneurysms. Bouzeghrane et al also concluded that the rabbit venous pouch bifurcation aneurysm model resulted so far in lower rates of aneurysm patency and higher rates of morbidity, making it less appropriate for embolic device evaluations as in, for example, the rabbit elastase model.
However, the rabbit venous pouch model could be substantially improved. ${ }^{4,5}$ It now offers equivalent patency and morbidity rates compared with the rabbit elastase and canine bifurcation models. Additionally, the unaltered vein transplant aneurysm walls of venous pouch bifurcation models show many similarities to the walls of human intracranial aneurysms. With proper technique, the foreign body reactions to the sutures are only located outside the aneurysms and, thus will not interfere with reactions to embolic devices. In contrast, the wall of elastase aneurysms consists of a former elastic artery, in which the multiple elastic membranes were destroyed by elastase, causing a long-lasting inflammatory repair process in the entire aneurysm wall.

Finally, because hemodynamics may be the most important factor leading to recanalization, the hemodynamic similarity of true bifurcation aneurysm models to human aneurysms with high rupture risks should be better considered in future experimental studies.

\section{References}

1. Bouzeghrane F, Naggara $O$, Kallmes $D$, et al. In vivo experimental intracranial aneurysm models: a systematic review. AJNR Am J Neuroradiol 2010;31: $418-23$

2. Sherif C, Plenk H Jr, Grossschmidt K, et al. Computer-assisted quantification of occlusion and coil densities on angiographic and histological images of experimental aneurysms. Neurosurgery 2006;58:559-66, discussion 59-66

3. Sherif C, Bavinzski G, Dorfer C, et al. Computerized assessment of angiographic occlusion rate and coil density in embolized human cerebral aneurysms. AJNR Am J Neuroradiol 2009;30:1046-53

4. Sherif C, Marbacher S, Erhardt S, et al. Improved microsurgical creation of venous pouch arterial bifurcation aneurysms in rabbits. AJNR Am J Neuroradiol. 2011;32:165-69

5. Marbacher S, Erhardt S, Schläppi A, et al. Complex bi-lobular, bi-saccular, and broad-necked microsurgical aneurysms formation in the rabbit bifurcation model for the study of upcoming endovascular techniques. AJNR Am J Neuroradiol (In press)

C. Sherif

Department of Neurosurgery Cantonal Hospital of Aarau

Aarau, Switzerland

H. Plenk, Jr

Department of Bone and Biomaterials Research Institute for Histology and Embryology Medical University of Vienna

Vienna, Austria

DOI 10.3174/ajnr.A2302 